\title{
Comparative transcriptome analysis of differentially expressed genes related to the physiological changes of yellow-green leaf mutant of maize
}

\author{
Tingchun Li ${ }^{\text {Corresp., } 1,2}$, Huaying Yang ${ }^{1}$, Yan Lu ${ }^{3}$, Qing Dong ${ }^{1}$, Guihu Liu ${ }^{1}$, Feng Chen ${ }^{2}$, Yingbing Zhou ${ }^{\text {Corresp. } 1}$ \\ ${ }^{1}$ Corn Research Center, Tobacco Research Institute, Anhui Academy of Agricultural Sciences, Hefei, China \\ 2 Department of Plant Sciences, University of Tennessee, Knoxville, United States \\ 3 Department of Biological Sciences, Western Michigan University, Kalamazoo, United States \\ Corresponding Authors: Tingchun Li, Yingbing Zhou \\ Email address: litingchun2003@126.com, ybzhou99@126.com
}

Chlorophylls, green pigments in chloroplasts, are essential for photosynthesis. Reduction in chlorophyll content may result in retarded growth, dwarfism, and sterility. In this study, a yellow-green leaf mutant of maize, indicative of abnormity in chlorophyll content, was identified. The physiological parameters of this mutant were measured. Next, global gene expression of this mutant was determined using transcriptome analysis and compared to that of wild-type maize plants. The yellow-green leaf mutant of maize was found to contain lower contents of chlorophyll $a$, chlorophyll $b$ and carotenoid compounds. It contained fewer active PSII centers and displayed lower values of original chlorophyll fluorescence parameters than the wild-type plants. The real-time fluorescence yield, the electron transport rate, and the net photosynthetic rate of the mutant plants showed reduction as well. In contrast, the maximum photochemical quantum yield of PSII of the mutant plants was similar to that of the wild-type plants. Comparative transcriptomic analysis of the mutant plants and wild-type plants led to the identification of differentially expressed 1122 genes, of which 536 genes were up-regulated and 586 genes down-regulated in the mutant. Five genes in chlorophyll metabolism pathway, nine genes in the tricarboxylic acid cycle and seven genes related to the conversion of sucrose to starch displayed downregulated expression. In contrast, genes encoding a photosystem II reaction center PsbP family protein and the PGR5-like protein 1A (PGRL1A) exhibited increased transcript abundance. 
1 Comparative transcriptome analysis of differentially expressed genes related to the physiological changes of

2 yellow-green leaf mutant of maize

3

4 Tingchun $\mathrm{Li}^{1}{ }^{1,2,}$, Huaying Yang ${ }^{1}$, Yan $\mathrm{Lu}^{3}$, Qing Dong ${ }^{1}$, Guihu Liu ${ }^{1}$, Feng Chen ${ }^{2}$, Yingbing Zhou ${ }^{1, *}$

51 Corn Research Center, Tobacco Research Institute, Anhui Academy of Agricultural Sciences, Hefei, 230031,

$6 \quad$ P.R.China;

72 Department of Plant Sciences, University of Tennessee, Knoxville, 37996;

83 Department of Biological Sciences, Western Michigan University, Kalamazoo, MI 49008

$9 *$ Corresponding author:

10 Tingchun Li and Yingbing Zhou

11 No. 40 South Nongke Road, Hefei, Anhui, 230031, China

12 Tel: +86-551-65148988.

13 E-mails: litingchun2003@126.com (Tingchun Li), yhy7646@sina.com (Huaying Yang), yan.1.lu@wmich.edu (Yan

14 Lu), dongqingiris@sina.com (Qing Dong), ycskygl@126.com (Guihu Liu), fengc@utk.edu (Feng Chen),

15 ybzhou99@126.com (Yingbing Zhou). 


\section{Abstract}

17 Chlorophylls, green pigments in chloroplasts, are essential for photosynthesis. Reduction in chlorophyll content may result in retarded growth, dwarfism, and sterility. In this study, a yellow-green leaf mutant of maize, indicative of abnormity in chlorophyll content, was identified. The physiological parameters of this mutant were measured. Next, global gene expression of this mutant was determined using transcriptome analysis and compared to that of wild-type maize plants. The yellow-green leaf mutant of maize was found to contain lower contents of chlorophyll $a$, chlorophyll $b$ and carotenoid compounds. It contained fewer active PSII centers and displayed lower values of original chlorophyll fluorescence parameters than the wild-type plants. The real-time fluorescence yield, the electron transport rate, and the net photosynthetic rate of the mutant plants showed reduction as well. In contrast, the maximum photochemical quantum yield of PSII of the mutant plants was similar to that of the wild-type plants. Comparative transcriptomic analysis of the mutant plants and wild-type plants led to the identification of differentially expressed 1122 genes, of which 536 genes were up-regulated and 586 genes down-regulated in the mutant. Five genes in chlorophyll metabolism pathway, nine genes in the tricarboxylic acid cycle and seven genes related to the conversion of sucrose to starch displayed down-regulated expression. In contrast, genes encoding a photosystem II reaction center PsbP family protein and the PGR5-like protein 1A (PGRL1A) exhibited increased transcript abundance.

Keywords: Yellow-green leaf; Chlorophyll biosynthesis; Photosynthesis; Tricarboxylic acid cycle; Secondary 
Chlorophylls are essential pigments for photosynthesis, playing the main role in the conversion of light energy to stored chemical energy (Gitelson et al., 2003). Chlorophyll content directly determine photosynthetic potential and primary productivity of green plants (Gitelson et al., 2003; Curran et al., 1990; Filella et al., 1995).The formation of chlorophyll consists of four steps including synthesis of 5-aminolevulinic acid, formation of a pyrrole ring porphobilinogen, synthesis of protoporphyrin IX and insertion of $\mathrm{Mg}^{2+}$ to the protoporphyrin IX (Wu et al., 2007). The functional genes of the chlorophyll metabolism pathway have been identified.

Generally, the leaf color is green for its common content of chlorophyll. Nevertheless, a large number of leaf color mutants have been identified in many seed plant species, such as Arabidopsis, maize, soybean, barley, rice, and wheat (Wang et al., 2018). Among the leaf color mutants, a number of abnormal phenotypes have been identified, such as yellow, pale green, spots, and stripes. Due to reduced levels of chlorophyll, retarded growth, dwarfism, and sterility were characterized in most color mutants. Recently, with the progressive characterization of various leaf color mutants, a significant number of genes have been isolated and verified to be responsible for the abnormal phenotype. For example, in carrot, a YEL locus was mapped in a linkage group with a total length of 33.2 $\mathrm{cM}$, and the mutant had a yellow-leaf phenotype (Nothnagel and Straka, 2003). In maize, a semi-dominant oil yellow $1(O y l)$ mutant was identified to be deficient in the conversion of protoporphyrin IX to magnesium protoporphyrin IX (Sawers et al., 2006). The Oyl gene was demonstrated to encode I subunit of magnesium chelatase (ZmCHLI). In cabbage, the $y g l-1$ locus was located on chromosome C01. Mutation in the $y g l-1$ gene exhibited a yellow-green leaf phenotype (Liu et al., 2016). In rice, mutation in the VIRESCENT YELLOW LEAF (VYL) gene, which encodes a subunit of chloroplast Clp (OsClpPO), resulted in temperature-insensitive and developmental stage-dependent virescent yellow leaf ( $v y l$ ) phenotype (Dong et al., 2013). Fgl is located in the coding region of OSPORB, and its mutation resulted in the presence of the yellow/white leaf (Sakuraba et al., 2019). Mutation in the $F d C 2$ gene, which encodes a ferredoxin-like protein with a C-terminal extension, caused the yellowgreen leaf phenotype in rice (Li et al., 2015). Mutation in the rice YS83 (LOC_Os02g05890) gene resulted in the yellow-green-leaf phenotype as well (Ma et al., 2017). In addition, a number of chloroplast signal recognition particle ( $c p S R P)$ mutants were identified with chlorophyll deficiency in Arabidopsis, rice and maize (Zhang et al., 2013; Guan et al., 2016; Wang et al., 2016). Although these studies have provided many insights into the key genes controlling chlorophyll deficiency, the analyses of physiological parameters of such mutants and their underlying molecular mechanisms have been generally lacking.

Chlorophyll-deficient mutants are important tools for studying the formation and development of photosynthetic pigments in plants. Their phenotypes could be used as crop trait markers in hybrid breeding (Zhong et al., 2015). In this work, a new yellow-green leaf mutant inbred line of maize was isolated. The chlorophyll content, chlorophyll 
69

fluorescence parameters, and photosynthesis characteristics were determined. Using comparative transcriptomic analysis, differentially expressed genes were compared between the yellow-green leaf mutant and the normal green leaf inbred line. These results not only provide valuable genetic resources for further studies of chlorophyll-deficient mutants in maize, but also contribute to our understanding of the relationship between physiological changes and gene expression changes. The latter may pave the way to further dissecting the molecular basis of morphological and physiological characteristics of the yellow-green leaf mutant.

\section{Materials \& Methods}

\section{Plant material}

The maize inbred line C033 and LH102 were obtained from preserved breeds in rural areas of Anhui province which were stored in Tobacco Research Institute, Anhui province, People's Republic of China. The yellow-green leaf mutant inbred line was isolated from an F2 segregating population of the recombination between inbred line C033 and LH102 in the farm of Tobacco Research Institute. After successive self-pollination of F2, F3 and F4 generations, a stable F5 generation was obtained. The yellow-green leaf mutant inbred line and a normal green leaf inbred line from the F5 generation were selected for downstream analyses. The inbred lines were cultivated with regular water and fertilizer management in the farm of Tobacco Research Institute in the year of 2017. After the third leaf was fully expanded, seedlings were selected for physiological parameter determination, RNA extraction, and gene expression analysis.

\section{Measurement of chlorophyll content}

Leaf samples $(100 \mathrm{mg}$ ) were cut into small pieces, and soaked in $10 \mathrm{~mL}$ of $80 \%$ acetone (acetone: water $=4: 1)$ at $4{ }^{\circ} \mathrm{C}$ in the dark for $24 \mathrm{~h}$. The supernatant was collected after centrifugation at $6000 \mathrm{rpm}$ for $10 \mathrm{~min}$. The absorbance was recorded at 663 and $645 \mathrm{~nm}$ on a UV-1800 spectrophotometer (Shimadzu Corporation, Japan). The concentrations of Chl $a$ and Chl $b$ were calculated using the method described by Arnon (1949). The values were calculated using three repeats.

\section{Measurement of carotenoid compounds}

To analyze the carotenoid compounds, $200 \mathrm{mg}$ leaf samples were grinded into powder with $2 \mathrm{ml}$ absolute alcohol containing $1 \%$ butylated hydroxytoluene. After water bath for $5 \mathrm{~min}$ at $85{ }^{\circ} \mathrm{C}, 40 \mathrm{ul} 80 \% \mathrm{KOH}$ and $1 \mathrm{ml} \mathrm{N}$-hexane were added into the extraction buffer followed by water bath and vortex. The supernatant were eventually collected and dried with nitrogen, then dissolved in $500 \mathrm{ul}$ acetonitrile solution containing $1 \%$ butylated hydroxytoluene, $25 \%$ methanol, and $5 \%$ dichloromethane for following analysis.

The Ultimate 3000 UHPLC system (Thermo Fisher Scientific, USA) was employed to quantitatively and qualitatively determine the components. Carotenoids were resolved and analyzed on a reverse phase YMC 
carotenoid column $\left(250 * 4.6 \mathrm{~mm}, 5 \mathrm{um}\right.$; YMC, Kyoto, Japan) set at a temperature of $40{ }^{\circ} \mathrm{C}$ with the flow rate of 1 $\mathrm{ml} \cdot \mathrm{min}^{-1}$. The solvent system consisted of solvent A with methanol: methyl tert-butyl ether: water (81:15:4, by vol) and solvent B with methanol: methyl tert-butyl ether (6.5:93.5, by vol). The gradient program was set as follows: 2 min hold on $100 \%$ solvent A, followed a 1 min linear gradient to $32.5 \%$ solvent $\mathrm{A}$ and $67.5 \%$ solvent $\mathrm{B}$, then 2 min hold on $100 \%$ solvent B, and 2 min hold on $100 \%$ solvent A lastly. Carotenoid compounds were detected at $450 \mathrm{~nm}$. The determination was repeated three times.

\section{Measurement of the total starch, total sugar and enzymes activities}

The contents of the total starch, the total soluble sugar and the total reducing sugar were measured using the methods described by Mccleary et al. (1994), Irigoyen et al. (1992) and Miller et al. (1959). The enzymes activities of SS (Sucrose synthase) and SPS (Sucrose phosphate synthase) were assayed according to Echeverria and Humphreys' method (Echeverria and Humphreys, 1985). The enzymes activities of SSS, GBSS and AGP were determined using the methods described by wang et al. (2007) and Nakamura et al. (1989). All the measurement of physiological parameters was repeated three times.

\section{Measurements of chlorophyll fluorescence parameters}

By using the PAM-2500 chlorophyll fluorometer (Walz, German), chlorophyll fluorescence parameters were determined for the leaves from the yellow-green leaf mutant inbred line and the normal green leaf inbred line. The procedure was described as follows. After the leaf was adapted in the dark with the dark adapting clip for 20 min, the slow kinetics of chlorophyll $a$ fluorescence induction was triggered with a continuous mode to measure darkand light-adapted parameters. The leaf was initially subjected to a measuring light of $95 \mu \mathrm{mol}$ photons $\cdot \mathrm{m}^{-2} \cdot \mathrm{s}^{-1}$. After Fo was recorded, a saturating pulse of $2000 \mu \mathrm{mol}$ photons $\cdot \mathrm{m}^{-2} \cdot \mathrm{s}^{-1}$ was automatically turned on, and Fm was measured accordingly. At that time, an actinic light of $145 \mu \mathrm{mol}$ photons $\cdot \mathrm{m}^{-2} \cdot \mathrm{s}^{-1}$ was activated to simulate normal irradiance conditions. F, Fm' and Fo' were subsequently measured with saturating pulses every $20 \mathrm{~s}$. The parameters $\Phi_{\mathrm{PSII}}, \Phi_{\mathrm{NPQ}}, \Phi_{\mathrm{NO}}, \mathrm{NPQ}, \mathrm{qN}, \mathrm{qP}, \mathrm{qL}$ and ETR were derived from the final measurements obtained after a 10 min light adaptation.

The maximum photochemical quantum yield of PSII (Fv/Fm) was calculated according to Stefanov and Terashima (2008): Fv/Fm $=(F m-F o) / F m$.

The effective photochemical quantum yield of PSII ( $\left.\Phi_{\mathrm{PSII}}\right)$, the quantum yield of non-regulated heat dissipation and fluorescence emission $\left(\Phi_{\mathrm{NO}}\right)$, and quantum yield of light-induced non-photochemical quenching $\left(\Phi_{\mathrm{NPQ}}\right)$ were calculated as described by Kramer et al. (2004): $\Phi_{\mathrm{PSII}}=\left(\mathrm{F}^{\prime} \mathrm{m}-\mathrm{F}\right) / \mathrm{F}^{\prime} \mathrm{m}, \Phi_{\mathrm{NO}}=1 /(\mathrm{NPQ}+1+\mathrm{qL}(\mathrm{Fm} / \mathrm{Fo}-1)), \Phi_{\mathrm{NPQ}}=$ $1-\Phi_{\mathrm{PSII}}-(1 /(\mathrm{NPQ}+1+\mathrm{qL}(\mathrm{Fm} / \mathrm{Fo}-1))$.

The coefficient of photochemical quenching ( $\mathrm{qL}$ ) was calculated as described by Kramer (2004): $\mathrm{qL}=\mathrm{qP} \times \mathrm{F}^{\prime} \mathrm{o} / \mathrm{F}$.

The coefficients of photochemical quenching (qP), non-photochemical quenching (qN and NPQ) were calculated 
132 as described by Stefanov and Terashima (2008): $\mathrm{qP}=\left(\mathrm{F}^{\prime} \mathrm{m}-\mathrm{F}\right) /\left(\mathrm{F}^{\prime} \mathrm{m}-\mathrm{Fo}\right), \mathrm{qN}=1-\left(\mathrm{Fm}-\mathrm{F}^{\prime} \mathrm{m}\right) /\left(\mathrm{Fm}-\mathrm{F}^{\prime} \mathrm{o}\right), \mathrm{NPQ}=(\mathrm{Fm}-$ $\left.133 \mathrm{~F}^{\prime} \mathrm{m}\right) / \mathrm{F}^{\prime} \mathrm{m}$.

134 The relative apparent photosynthetic electron transport rate $(\mathrm{ETR})$ was calculated using the equation: ETR $=$

135

136

137 138

140

141

142

143

144

145

146

147

148

149

150

151

152

153

154

155

156

157 158

159

160

161

162 163 $\Phi_{\mathrm{PSII}} \times \mathrm{PAR} \times 0.5 \times 0.84$

\section{Measurement of photosynthesis parameters}

The net photosynthetic rate (Pn) was measured using a Li-6400XT portable photosynthesis system (Li-Cor Inc., USA) equipped with a 6400-02B chamber and a red-blue LED light source with intensities up to $2000 \mu \mathrm{mol}$ photons $\cdot \mathrm{m}^{-2} \cdot \mathrm{s}^{-1}$ over an area of $6 \mathrm{~cm}^{2}$. The flow rate was adjusted to $500 \mu \mathrm{mol} \cdot \mathrm{s}^{-1}$ with the absolute $\mathrm{CO}_{2}$ concentration of $380 \mu \mathrm{mol} \cdot \mathrm{mol}^{-1}$ at $26{ }^{\circ} \mathrm{C}$ inside the chamber. The light response curve of Pn was determined at nine photosynthetically active radiation $(\mathrm{PAR})$ levels $\left(0,50,100,200,400,800,1200,1600\right.$ and $2000 \mu \mathrm{mol} \mathrm{photons}^{\cdot \mathrm{m}^{-}}$ $\left.{ }^{2} \cdot \mathrm{s}^{-1}\right)$. Three biological repeats were created.

\section{cDNA library construction and sequencing}

By using Illumina TruSeq ${ }^{\text {TM }}$ RNA Sample Preparation Kit (Illumina, San Diego, USA), the cDNA library was constructed. After the quality detection, the Illumina sequencing was carried out at Beijing Novogene Biological Information Technology Co. Ltd. (Beijing, China) (http://www.novogene.cn/). The index-coded samples were clustered following the manufacturer's instructions using TruSeq PE Cluster Kit v3-cBot-HS (Illumina). Then, the library was sequenced to generate 200 bp paired-end reads on an Illumina Hiseq 2500 platform. The raw data was accessible at the Sequence Read Archive Database of NCBI (https://www.ncbi.nlm.nih.gov/) with the accession number PRJNA629016.

\section{Data filtering and assembly}

The clean reads were obtained after remove of duplicated sequences, ploy-N, adaptor sequences and low-quality reads. Then, they were aligned to the the maize B73 reference genome (AGPv4) using TopHat 2 as previously described (Schnable et al., 2009; Kim et al., 2013). The resulting read counts were normalized by per kilobase million mapped reads (RPKM) to measure the gene expression level (Mortazavi et al., 2008). Maize reference genome sequence data were downloaded at ftp://ftp.ncbi.nlm.nih.gov/genomes/genbank/plant/Zea_mays/latest_assembly_versions/GCA_000005005.6_B73_Re fGen_v4.

\section{Identification of differential expressed genes}

To identify differentially expressed gene (DEGs) in the comparison settings, the raw counts were imported into the edgeR and adjusted with one scaling normalized factor (Zhang et al., 2014). Then the DEGseq R package (1.12.0; TNLIST, Beijing, China) was employed to screen out the DEGs with $P$-value $<0.05$ (Benjamini and Hochberg, 1995). The sequences of all DEGs were listed in Table S6. 
164 Gene functional annotation and metabolic pathway analysis

165 The DEGs were annotated via alignment against and comparison with Pfam (http://pfam.xfam.org/), NCBI non-

166 redundant $(\mathrm{Nr})$ protein database (ftp://ftp.ncbi.nih.gov/blast/db/),), and SwissProt protein database

167 (https://web.expasy.org/docs/swiss-prot guideline.html). The GO terms including molecular function, biological

168 process, and cellular component ontology were analyzed using Blast2GO program. Pathway assignments were

169 conducted according to the Kyoto Encyclopedia of Genes and Genomes Pathway database (KEGG

170 http://www.genome.jp/kegg). To map the target genes to metabolic pathways, all sequences of DEGs were uploaded

171 to the Mercator v.3.6 (https://www.plabipd.de/portal/web/guest/mercator-sequence-annotation) to generate root map

172 file, then it was imported to the Mapman software (V3.6.0 RC1) to obtain the map based on the transcriptome data.

173 Verification of unigenes and gene expression profiling using RT-qPCR

174 Quantitative Real-Time PCR was performed to quantify nine DEGS to evaluate the validity of transcriptome data.

175 The candidate genes and their primers are listed in Supplementary Table 1. The RNAprep Pure Plant Kit (Tiangen,

176 China) was used to obtain the total RNA. The PCR system contained $2 \mu$ primers, $2 \mu 1$ of $\mathrm{cDNA} 8.5 \mu \mathrm{l}$ of ddH ${ }_{2} \mathrm{O}$,

177 and $12.5 \mu \mathrm{l}$ of SYBR ${ }^{\circ}$ Premix Ex TaqTM II. PCR amplifications follow the procedure, $95{ }^{\circ} \mathrm{C}$ for $30 \mathrm{~s}, 95{ }^{\circ} \mathrm{C}$ for $5 \mathrm{~s}$,

$17860{ }^{\circ} \mathrm{C}$ for $30 \mathrm{~s}, 40$ cycles. Quantification was calculated with the $2^{-\Delta \Delta \mathrm{Ct}}$ method (Livak et al., 2001).

179 Results

180 Identification of a maize yellow-green leaf mutant and measurement of its pigment contents

181 A yellow-green leaf mutant inbred line was firstly isolated from an F2 segregating population of the cross recombination inbred line $\mathrm{C} 033$ and inbred line LH102, both of which have a green leaf phenotype. After successive 183 self-pollination of F2, F3 and F4 generations, a stable F5 generation was obtained. In this study, a stable yellowgreen leaf mutant inbred line and a normal green leaf inbred line from the F5 generation were selected for downstream characterizations. The yellow-green leaf mutant had a yellow color in the entire above-ground portion of the plant (Fig. 1A).

Leaf color could indicate the amount and proportion of chlorophyll in leaves. Deficiency of chlorophyll leads to the leaf color change from green to yellow. In this study, in contrast with the normal green leaf inbred line, the content of chlorophyll $a$ and chlorophyll $b$ in the yellow-green leaf mutant was reduced by $35.22 \%$ and $34.48 \%$, respectively, which may directly result in the presence of yellow-green color in the mutant plants (Fig. 1B).

191 Otherwise, the contents of seven kinds of carotenoid compounds including neoxanthin, violaxanthin, capsanthin, zeaxanthin, $a$-carotene, $\beta$-carotene and lutein were significantly decreased in the yellow-green leaf mutant (Fig. 1C). 
absorption and energy transfer in the light-harvesting complexes. Fo indicates the minimum fluorescence yield after dark-adaptation with all PSII centers open. Fm represents the maximum fluorescence yield after dark-adaptation with all PSII centers closed. Both Fo and Fm were decreased in the yellow-green leaf mutant, suggesting that the yellow-green leaf mutant has fewer active PSII centers than the normal green leaf inbred line (Fig. 2A). However, the yellow-green leaf mutant and the normal green leaf inbred line had the similar value of the maximum photochemical quantum yield of PSII (Fv/Fm), suggesting that light absorption and energy transfer of the lightharvesting complexes is still efficient in the yellow-green leaf mutant plants.

Ft is the real-time fluorescence yield recorded during the slow kinetics induction with the continuous monitoring mode. The changes of Ft reflect the light-adaption status of the PSII centers. Although the Ft kinetics curve in the yellow-green leaf mutant had the same light-adaption pattern as that in the normal green leaf inbred line, the Ft values were much lower in the yellow-green leaf mutant (Fig. 2B). Accordingly, the values of the minimal and maximal fluorescence yield in the light-adapted state (Fo' and Fm') were significantly lower in the yellow-green leaf mutant than in the normal green leaf inbred line (Fig. $2 \mathrm{C}$ ). Photochemical quenching parameters ( $\Phi_{\mathrm{PSII}}$, $\mathrm{qP}$, and qL), non-photochemical quenching parameters ( $\Phi_{\mathrm{NO}}, \Phi_{\mathrm{NPQ}}, \mathrm{NPQ}$, and $\left.\mathrm{qN}\right)$, and the PSII electron transport rate (ETR) were also evaluated. The values of $\Phi_{\mathrm{PSII}}, \mathrm{qP}, \mathrm{qL}, \Phi_{\mathrm{NO}}, \Phi_{\mathrm{NPQ}}, \mathrm{NPQ}$, and $\mathrm{qN}$ were similar between the yellowgreen leaf mutant and the normal green leaf inbred line (Fig. 2C). But the value of ETR was significantly lowered in the yellow-green leaf mutant.

\section{Net photosynthesis in response to light intensities of the yellow-green leaf mutant}

Net photosynthesis (Pn) in response to different light intensities was also determined (Fig. 2D). Pn was lower in the yellow-green leaf mutant than in the normal green leaf inbred line when the light intensity was $1200 \mu \mathrm{mol}$ photons $\cdot \mathrm{m}^{-2} \cdot \mathrm{s}^{-1}$ or higher. But the rate of dark respiration was higher in the yellow-green leaf mutant.

\section{Comparative transcriptomic analysis of the yellow-green leaf mutant and the normal green leaf inbred line:} overall changes

To explain the physiological changes in the yellow-green leaf mutant, comparative transcriptome analysis was performed to identify differentially expressed genes in chlorophyll biosynthesis, light-harvesting antenna complex formation, photosynthesis, and other metabolism pathways. Three independently repeated sequencing of cDNA was carried out by using the HiSeq 2500 platform. After data processing, the clean reads were obtained. Pearson correlation was calculated for the three independent experiments. The data from NGL_1, NGL_3, YGL_2, and YGL_3 were collected for the following analysis for the higher correlation rate between the same color leaves (Fig. S1). Further studies were performed to identify differentially expressed genes, using DGE methods. A total of 1122 genes were found to be differentially expressed in the normal green leaf inbred line and the yellow-green leaf mutant: 536 genes were up-regulated, and 586 genes were down-regulated in the yellow-green leaf mutant (Fig. 3A and 
Table S2). Most of these genes were enriched in biological processes and molecular functions (Table S3). Based on the KEGG pathway analysis and pathway assignment of MapMan, of all these differentially expressed genes, 1092 genes were mapped to 33 metabolic pathways, including photosynthesis (14 genes), lipid metabolism (40 genes), secondary metabolism (52), stress response (55), and so on (Fig. 3B, Table S3 and Table S4). The remaining 328 genes were not assigned.

\section{Differentially expressed genes in chlorophyll biosynthetic pathways}

Chlorophylls are synthesized from the precursor protophorphyrin IX. As shown in Fig. 4, three chlorophyll metabolic genes (magnesium-chelatase subunit chlD [Zm00001d013013], protochlorophyllide reductase A [Zm00001d001820], and chlorophyllide a oxygenase [Zm00001d004531] showed decreased expression levels in the yellow-green leaf mutant. Similarly, two protophorphyrin biosynthetic genes (coproporphyrinogen III oxidase [Zm00001d026277] and uroporphyrinogen decarboxylase 1 [Zm00001d044321]) displayed decreased expression in the yellow-green leaf mutant. However, genes encoding chlorophyllide $a$ oxygenase (Zm00001d002358) and

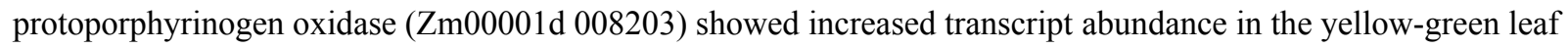
mutant.

\section{Differentially expressed genes in photosynthetic reactions}

As shown in Fig. 5, 22 genes in photosynthesis were differentially expressed in the yellow-green leaf mutant and the normal green leaf inbred line. Of these genes, two are related to photosynthetic light reactions, 11 genes participate in photorespiration, and nine are involved in the Calvin cycle. Among the two genes in photosynthetic light reactions, one encodes a Mog1/PsbP/DUF1795-like photosystem II reaction center PsbP family protein (Zm00001d041824) and the other encodes PGRL1A (PGR5-like protein 1A, Zm00001d034904). These two genes exhibited increased transcript abundance in the yellow-green leaf mutant. However, genes encoding two ribulose bisphosphate carboxylase/oxygenase (RuBisCO) large chain precursors (Zm00001d00279 and GRMZM5G815453), which are involved in the Calvin cycle and photorespiration, showed decreased transcript abundance in the yellowgreen leaf mutant. Genes encoding two RuBisCO large subunit-binding protein subunit alpha (Zm00001d031503 and Zm00001d051252) displayed decreased transcript abundance in the yellow-green leaf mutant as well. Genes encoding chloroplast chaperonin 60 subunit beta (Zm00001d035937), RuBisCO methyltransferase family protein (Zm00001d020437), TCP/cpn60 chaperonin family protein (Zm00001d045544), and RuBisCO large subunitbinding protein subunit alpha (Zm00001d00399) showed increased transcript abundancein the yellow-green leaf mutant. The expression of phosphoglycolate phosphatase (Zm00001d034887) and glycerate dehydrogenase (Zm00001d014919) was enhanced in the yellow-green leaf mutant, whereas the expression of glycine dehydrogenase (Zm00001d023437) and aldolase superfamily protein (Zm00001d040084) was decreased.

\section{Differentially expressed genes in the tricarboxylic acid cycle}


259 The tricarboxylic acid cycle (TCA cycle) is responsible for the production of most of the ATP yield. As shown in

260 Fig. 6, a total of nine genes in the TCA cycle were down-regulated in the yellow-green leaf mutant. Among these 261 genes, pyruvate phosphate dikinase (Zm00001d010321) catalyzes the conversion of pyruvate to

262 phosphoenolpyruvate (PEP), aconitatehydratase (Zm00001d015497) catalyzes the stereo-specific isomerization of 263 citrate to isocitrate, and isocitrate dehydrogense (Zm00001d025690) catalyzes the conversion of isocitrate to alpha-

264 ketogutarate and $\mathrm{CO}_{2}$. The other six genes are involved in oxidative phosphorylation: NADH-ubiquinone

265 oxidoreductase $20 \mathrm{kDa}$ subunit (Zm00001d043619), NADH dehydrogenase (Zm00001d016864), ubiquinol-

266 cytochrome c reductase iron-sulfur subunit (Zm00001d016619), cytochrome c (Zm00001d042600), member of

267 uncoupling protein PUMP2 family (Zm00001d048583), and cytochrome c oxidase (Zm00001d051055).

\section{Differentially expressed genes in the sucrose-to-starch pathway}

269 In Fig. 7, nine genes in the sucrose-to-starch pathway were found to be differentially expressed between the yellow270 green leaf mutant and the normal green leaf inbred line. Among these genes, two were up-regulated and seven were 271 down-regulated in the yellow-green leaf mutant. Among the two up-regulated genes, one encodes aglycosyl

272 hydrolase family 32 protein (Zm00001d025943), which may function as the invertase that split sucrose into glucose 273 and fructose. The other gene is annotated as fructokinase-like protein (Zm00001d033181), which may catalyze the 274 conversion of fructose to fructose-6- phosphate. Among the seven down-regulated genes, three are annotated as 275 granule-bound starch synthase 1b (Zm00001d027242, Zm00001d029360, and Zm00001d019479), two encode 276 soluble starch synthase (Zm00001d0002256 and Zm00001d0045261) and one encodes 1, 4-alpha-glucan branching 277 enzyme IIB (Zm00001d003817). These six genes participate in starch synthesis. The other down-regulated gene is 278 annotated as alpha-1, 4 glucanphosphrylase L isozyme (Zm00001d034074), which catalyzes the conversion of 279 starch to glucose-1-phosphate.

\section{Validation of unigenes and gene expression profiling}

281 Nine candidate genes were selected to test the validity of transcriptome date using QRT-PCR. The results showed 282 that the expression patterns of nine genes determined using RT-qPCR were consistent with the transcriptome data, 283 indicating the transcriptome data were very reliable (Additional file: Fig. S4).

\section{Discussion}

285 In maize, a yellow-green leaf mutant SN62 has been identified (Zhong et al., 2015). Photosynthetic characteristics of 286 SN62 revealed that its chlorophyll content, the quantum efficiency of PSII and maximal quantum yield of PSII 287 photochemistry were significantly lower than those of a medium-green leaf inbred line SN12 (Zhong et al., 2015). In 288 this study, another yellow-green leaf mutant was identified. The values of photosynthetic parameters in this newly 289 identified yellow-green leaf mutant were comparable to those in the previously identified yellow-green leaf mutant 
290 (SN62). The chlorophyll content and the values of chlorophyll fluorescence parameters (Ft, Fo, Fm, Fo', Fm' and

291 ETR) in the yellow-green leaf mutant were significantly lowered than those in the normal green leaf inbred line.

292 These data indicate that the yellow-green leaf mutant has fewer opened PSII reaction centers than the normal green

293 leaf inbred line. However, the yellow-green leaf mutant and the normal green leaf inbred line had the similar value

294 of the maximum photochemical quantum yield of PSII (Fv/Fm). This suggests that light absorption and energy

295 transfer of the light-harvesting complexes is still efficient in the yellow-green leaf mutant plants. In addition, there

296 were no obvious differences in $\Phi_{\mathrm{PSII}}, \Phi_{\mathrm{NO}}, \Phi_{\mathrm{NPQ}}, \mathrm{NPQ}, \mathrm{qN}, \mathrm{qP}$, and qL between the yellow-green leaf mutant and

297 the normal green leaf inbred line. Furthermore, Pn was only lower in the yellow-green leaf mutant than in the

298 normal green leaf inbred line when the light intensity was at $1200 \mu \mathrm{mol}$ photons $\cdot \mathrm{m}^{-2} \cdot \mathrm{s}^{-1}$ or higher.

299 In seed plants, most of the genes responsible for the chlorophyll biosynthesis pathway have been identified

300 (Tripathy and Pattanayak, 2012). Magnesium-protoporphyrin chelatase catalyzes the first step in chlorophyll

301 synthesis. This enzyme contains three subunits (ChlH, ChlD and ChlI) and catalyzes the insertion of $\mathrm{Mg}^{2+}$ into

302 protoporphyrin IX. Mutation in ChlD resulted in a chlorina (yellowish-green) phenotype in rice (Zhang et al., 2006).

303 Protochlorophyllide reductase catalyzes the conversion of pchlide to chloro-phyllide. Chlorophyllide $a$ oxygenase

$304(\mathrm{CAO})$ is responsible for chlorophyll $b$ biosynthesis (Reinbothe et al., 2006). Overexpression of CAO was found to 305 enlarge the antenna size of photosystem II in Arabidopsis (Tanaka et al., 2001). Mutation in the barley CAO (fch2) 306 gene leads to chlorophyll $b$ deficiency, which may affect electron transfer in photosystem II (Mueller et al., 2012).

307 Coproporphyrinogen III oxidase is a key enzyme in the biosynthetic pathway of chlorophyll and heme. The

308 deficiency in coproporphyrinogen III oxidase caused lesion formation in Arabidopsis (Ishikawa et al., 2001).

309 Uroporphyrinogen decarboxylaseis is responsible for the decarboxylation of four acetate groups of

310 uroporphyrinogen III to yield coproporphyrinogen III, resulting in heme and chlorophyll biosynthesis (Fan et al.,

311 2007). In this study, these genes were found to have decreased transcript abundance in the yellow-green leaf mutant,

312 which may directly cause chlorophyll deficiency and reduced formation of light-harvesting antenna complexes.

313 Photosynthesis begins with the light reactions. In this work, the gene encoding Mog1/PsbP/DUF1795-like

314 photosystem II reaction center PsbP family protein and the gene annotated as PGRL1A were found to have

315 increased transcript abundance. PsbP is necessary for the retention of $\mathrm{Ca}^{2+}$ and $\mathrm{Cl}^{-1}$, the assembly of PSII complex,

316 and the maintenance of normal thylakoid architecture in PSII (Cao et al., 2015). PGRL1A is associated with PSI and

317 it interacts with PGRL1 (DalCorso et al., 2008). The PGRL1-PGR5 complex was found to facilitate cyclic electron

318 flow (DalCorso et al., 2008). In this work, the expression of both genes was up-regulated in the yellow-green leaf

319 mutant, which may make the fewer opened PSII reaction centers work efficiently in the yellow-green leaf mutant.

320 TCA cycle plays a central role in generating ATP and providing reducing agent NADH and precursors for a 321 number of amino acids in both heterotrophic and photosynthetic tissues (Daloso et al., 2015). In this work, totally

Peer] reviewing PDF | (2020:06:49778:3:0:NEW 6 Nov 2020) 
322

323

324

325

326

327

328

329

330

331

332

333

334

335

336

337

338

339

340

\section{Conclusions}

342

nine genes were identified with down-regulated expression in the yellow-green leaf mutant, including pyruvatephosphate dikinase, aconitatehydratase, isocitrate dehydrogenase, $\mathrm{NADH}$ dehydrogenase, and $\mathrm{NADH}$ ubiquinone oxidoreductase $20 \mathrm{kDa}$ subunit. Pyruvate phosphate dikinase is the key enzyme in cellular energy metabolism; it catalyzes the ATP- and phosphate $\left(\mathrm{P}_{\mathrm{i}}\right)$-dependent conversion of pyruvate to phosphoenol pyruvate in C4 plants (Ciupka and Gohlke., 2017). Aconitate hydratase catalyzes the conversion of citrate to cis-aconitate (Lichardusova et al., 2017). Isocitrate dehydrogenase catalyzes oxidative decarboxylation of isocitrate (Mhamdi and Noctor, 2015). The other six genes are involved in the mitochondrial electron transport chain and ATP synthesis, which requires the participation of large protein complex I (NADH-ubiquinone oxidoreductase), II (NADH dehydrogenase), III (ubiquinol-cytochrome $c$ reductase) and IV (cytochrome $c$ oxidase) (Møller, 2001; Dudkina et al., 2005). The decreased expression of these genes may have negative effects on ATP generation in the mitochondria of the yellow-green leaf mutant.

Furthermore, seven genes involved in the conversion of sucrose to starch were found to have decreased transcript abundance in the yellow-green leaf mutant. Of these genes, granule-bound starch synthase $1 \mathrm{~b}$ is responsible for amylose synthesis (Suzuki et al., 2015). Soluble starch synthase is a key enzyme in the biosynthesis of amylopectin (Wang et al., 2017). Moreover, the analysis of enzymes activities in starch biosynthesis pathway further demonstrated the results. The enzymes activities of SS, SSS, SPS and GBSS were significantly lower in yellowgreen leaf if compared with that in normal green leaf (Additional file: Fig. S2). But there were no obvious difference in the contents of starch and total reducing sugar, though the content of the water-soluble total sugar was high in normal green leaf (Additional file: Fig. S3).

In summary, the yellow-green mutant leaf was identified with obviously lowered chlorophyll content. The phenotype changes directly caused the decrease of light absorption and energy transfer, photosynthesis, and starch synthesis. Comparative transcriptome analysis identified the differentially expressed gene between the yellow-green leaf and normal green leaf. Further analysis revealed that the changes of genes expression were consistent with the variation of physiological data. The downregulated expression of genes in chlorophyll biosynthesis pathway resulted in chlorophyll deficiency in yellow-green mutant leaf. Then, it negatively affected the expression of genes in photosynthesis, TCA cycle, starch biosynthesis, and so on. The enzymes activities, the net photosynthesis rate, and water-soluble sugar were eventually decreased in yellow-green mutant leaf. These findings provide potential explanations for observed morphological and physiological changes in the yellow-green leaf mutant. Further investigations are needed to unravel the molecular basis of the morphological, physiological and transcriptional changes in the yellow-green leaf mutant plants. 
353

354

355

356

357

358

359

360

361

362

363

364

365

366

367

368

369

370

371

372

373

374

375

376

377

378

379

\section{Author Contributions}

Tingchun Li and Huaying Yang performed experiments, generated figures, and wrote the draft manuscript. Yan Lu helped with data interpretation and revision of the manuscript. Qing Dong and Guihu Liu helped in performing experiments and preparation of the materials. Feng Chen helped with data interpretation and revision of the manuscript. Yingbing Zhou designed the plan and significantly revised the manuscript. All authors have read and approved the final manuscript.

\section{Additional Information}

\section{Supplementary Materials:}

Table S1 Primers for qRT-PCR analysis

Table S2 The list of differentially expressed genes between yellow-green leaf and normal green leaf

Table S3 GO terms analysis of differentially expressed genes

Table S4 KEGG pathway analysis of differentially expressed genes

Table S5 Pathway assignment of differentially expressed genes using MapMan software

Table S6 The sequences of all differentially expressed genes

Fig. S1 The Pearson correlation between individual RNA samples from the yellow-green leaf mutant and the normal green leaf inbred line. The $\mathrm{R}^{2}$ value of the Pearson correlation between each pair of samples is presented in the center of each square. NGL_1, NGL_2 and NGL_3 are the three repetitions of the normal green leaf inbred line. YGL_1, YGL_2 and YGL_3 are the three repetitions of yellow-green leaf mutant.

Fig. S2 The activities of enzymes SS, SSS, SPS, GBSS and AGP involved in starch biosynthesis pathway. Small letters $\mathrm{a}$ and $\mathrm{b}$ above the columns indicate differences between the yellow-green leaf mutant and the normal green leaf inbred line at $P<0.05$, according to least significant difference (LSD) tests

Fig. S3 The contents of starch, total reducing sugar and the water-soluble total sugar

Fig. S4 Validation of unigenes and DGE genes expression profiling

\section{References}

Arnon DI. 1949. Copper enzymes in isolated chloroplasts. Polyphenoloxidase in Beta vulgaris. Plant Physiology 24:

Benjamini Y, Hochberg Y. 1995. Controlling the false discovery rate: a practical and powerful approach to multiple testing. Journal of the Royal Statistical Society. Series B (Methodological) 57:289-300

Cao P, Xie Y, Li M, Pan XW, Zhang HM, Zhao XL, Su XD, Cheng T, Chang W. 2015. Crystal structure analysis of extrinsic PsbP protein of photosystem II reveals a manganese-induced conformational change. Molecular Plant 8:664-666.

Ciupka D, Gohlke H. 2017. On the potential alternate binding change mechanism in a dimeric structure of Pyruvate 
Phosphate Dikinase. Scientific Reports 7:8020.

Curran PJ, Dungan JL, Gholz HL. 1990. Exploring the relationship between reflectance red edge and chlorophyll content in slash pine leaves. Tree Physiology 15:33 -48

DalCorso G, Pesaresi P, Masiero S, Aseeva E, Schünemann D, Finazzi G, Joliot P, Barbato R, Leister D. 2008. A complex containing PGRL1 and PGR5 is involved in the switch between linear and cyclic electron flow in Arabidopsis. Cell 132:273-285.

Daloso DM, Müller K, Obata T, Florian A, Tohge T, Bottcher A, Riondet C, Bariat L, Carrari F, Nunes-Nesi A, Buchanan BB, Reichheld JP, Arujo WL, Fernie AR. 2015. Thioredoxin, a master regulator of the tricarboxylic acid cycle in plant mitochondria. Proceedins of the National Academy of Sciences of the United States of America 112:E1392-E1400.

Dong H, Fei GL, Wu CY, Wu FQ, Sun YY, Chen MJ, Ren YL, Zhou KN, Cheng ZJ, Wang JL, Jiang L, Zhang X, Guo XP, Lei CL, Su N, Wang HY, Wan JM. 2013. A rice virescent-yellow leaf mutant reveals new insights into the role and assembly of plastid caseinolytic protease in higher plants. Plant Physiology 162:1867-1880.

Dudkina NV, Eubel H, Keegstra W, Boekema EJ, Braun HP. 2005. Structure of a mitochondrial supercomplex formed by respiratory-chain complexes I and III. Proceedins of the National Academy of Sciences of the United States of America 12:3225-3229

Echeverria E, Humphreys T. 1985. Glucose control of sucrose synthase in the maize scutellum. Phytochemistry 24:2851-2855

Gitelson AA, Gritz Y, Merzlyak MN. 2003. Relationships between leaf chlorophyll content and spectral reflectance and algorithms for non-destructive chlorophyll assessment in higher plant leaves. Journal of Plant Physiology 160:271-282

Guan H, Xu X, He C, Liu C, Liu Q, Dong R, Liu T, Wang L. 2016. Fine mapping and candidate gene analysis of the Leaf-Color Gene ygl-lin maize. PLoS One 11:e0153962.

Fan J, Liu Q, Hao Q, Teng M, Niu L. 2007. Crystal structure of uroporphyrinogen decarboxylase from Bacillus subtilis. Journal of Bacteriology 189:3573-3580.

Filella I, Serrano I, Serra J, Peñuelas J. 1995. Evaluating wheat nitrogen status with canopy relfectance indices and discriminant analysis. Crop Science 35:1400-1405

Irigoyen JJ, Emerich DW, Sanchez-Diaz M. 1992. Water stress induced changes in concentrations of proline and total soluble sugars in nodulated alfalfa (Medicago sativa) plants. Physiologia Plantarum 84:55-60.

Ishikawa A, Okamoto H, Iwasaki Y, Asahi T. 2001. A deficiency of coproporphyrinogen III oxidase causes lesion formation in Arabidopsis. The Plant Journal 27:89-99.

Kakumanu A, Ambavaram MM, Klumas C, Krishnan A, Batlang U, Myers E, Grene R, Pereira, A. 2012. Effects of 
417

drought on gene expression in maize reproductive and leaf meristem tissue revealed by RNA-Seq. Plant Physiology 160:846-867

Kim DC, Pertea G, Trapnell C, Pimentel H, Kelley R, Salzberg SL. 2013. TopHat2: accurate alignment of transcriptomes in the presence of insertions, deletions and gene fusions. Genome Biology 14:R36.

Kramer DM, Johnson G, Kiirats O, Edwards GE. 2004. New fluorescence parameters for the determination of QA redox state and excitation energy fluxes. Photosynthesis Research 79:209.

Li C, Hu Y, Huang R, Ma X, Wang Y, Liao T, Zhong P, Xiao F, Sun C, Xu Z, Deng X, Wang P. 2015. Mutation of FdC2 gene encoding a ferredoxin-like protein with C-terminal extension causes yellow-green leaf phenotype in rice. Plant Science 238:127-134.

Lichardusova L, Tatarkova Z, Calkovska A, Mokra D, Engler I, Racay P, Lehotsky J, Kaplan P. 2017. Proteomic analysis of mitochondrial proteins in the guinea pig heart following long-term normobarichyperoxia. Molecular and Cellular Biochemistry 434:61-73.

Liu XP, Yang C, Han FQ, Fang ZY, Yang LM, Zhuang M, Lv HH, Liu YM, Li ZS, Zhang YY. 2016. Genetics and fine mapping of a yellow-green leaf gene ( $y g l-1)$ in cabbage (Brassica oleracea var. capitata L.). Molecular Breeding 36:82.

Livak KJ, Schmittgen TD. 2001. Analysis of relative gene expression data using real-time quantitative PCR and the 2(-Delta Delta C(T)) Method. Methods 25, 402-408.

Ma X, Sun X, Li C, Huan R, Sun C, Wang Y, Xiao F, Wang Q, Chen P, Ma F, Zhang K, Wang P, Deng X. 2017. Map-based cloning and characterization of the novel yellow-green leaf gene ys 83 in rice (Oryza sativa). Plant Physiology and Biochemistry 111: 1-9.

Mccleary BV, Giboson TS, Solah V, Mugford DC. 1994. Total starch measurement in cereal products: interlaboratory evaluation of a rapid enzymic test procedure. Cereal Chemistry 71:501-504.

Mhamdi A, Noctor G. 2015. Analysis of the roles of the Arabidopsis peroxisomal isocitrate dehydrogenase in leaf metabolism and oxidative stress. Environmental and Experimental Botany 114:22-29.

Miller GL. 1959. Use of dinitrosalicylic acid reagent for determination of reducing sugar. Analytical Chemistry

$$
31: 426-428 .
$$

Mueller AH, Dockter C, Gough SP, Lundqvist U, von Wettstein D, Hansson M. 2012. Characterization of mutations in barley fch2 encoding chlorophyllidea oxygenase. Plant and Cell Physiology 53:1232-1246.

Møller IM. 2002. A new dawn for plant mitochondrial NAD(P)H dehydrogenases. Trends in Plant Science 7:235237.

Mortazavi A, Williams BA, McCue K, Schaeffer L, Wold B. 2008. Mapping and quantifying mammalian transcriptomes by RNA-Seq. Nature Methods 5:621. 
Nakamura Y, Yuki Y, Park SY. 1989. Carbohydrate metabolism in the developing endo-sperm of rice grains. Plant and Cell Physiology 30:833-839.

Nothnagel T, Straka P. 2003. Inheritance and mapping of a yellow leaf mutant of carrot (Daucuscarota). Plant Breeding 122:339-342.

Reinbothe C, Bartsch S, Eggink LL, Hoober JK, Brusslan J, Andrade-Paz R, Monnet J, Reinbothe S. 2006. A role for chlorophyllideaoxygenase in the regulated import and stabilization of light-harvesting chlorophyll $\mathrm{a} / \mathrm{b}$ proteins. Proceedins of the National Academy of Sciences of the United States of America 103:4777-4782.

Sakuraba Y, Rahman ML, Cho SH, Kim YS, Koh HJ, Yoo SC, Paek NC. 2013. The rice faded green leaf locus encodes protochlorophyllideoxido reductase B and is essential for chlorophyll synthesis under high light conditions. The Plant Journal 74:122-133.

Sawers RJ, Viney J, Farmer PR, Bussey RR, Olsefski G, Anufrikova K, Neil Hunter C, Brutnell TP. 2006. The maize Oil yellowl (Oyl) gene encodes the I subunit of magnesium chelatase. Plant Molecular Biology 60:95106.

Schnable PS, Ware D, Fulton R, Stein JC, Wei F, Pasternak S, et al. 2009. The B73 maize genome. Science 326:1112-1115.

Stefanov D, Terashima I. 2008. Non-photochemical loss in PSII in high- and low-light-grown leaves of Vicia faba quantified by several fluorescence parameters including $\mathrm{L}_{\mathrm{NP}}$, Fo/Fm', a novel parameter. Physiology Plantarum 133:327-338.

Suzuki Y, Arae T, Green PJ, Yamaguchi J, ChibaY. 2015. AtCCR4a and AtCCR4b are involved in determining the poly (A) length of granule-bound starch synthase 1 transcript and modulating sucrose and starch metabolism in Arabidopsis thaliana. Plant and Cell Physiololgy 56:863-874.

Tanaka R, Koshino Y, Sawa S, Ishiguro S, Okada K, Tanaka A. 2001. Overexpression of chlorophyllide a oxygenase (CAO) enlarges the antenna size of photosystem II in Arabidopsis thaliana. The Plant Journal 26:365-373.

Tripathy BC, Pattanayak GK. 2012. Chlorophyll biosynthesis in higher plants. In Photosynthesis. Dordrecht: Springer; p. 63-94.

Wang F, Chen S, Cheng F, Liu Y, Zhang G. 2007. The differences in grain weight and quality within a rice (Oryza sativa L.) panicle as affected by panicle type and source sink relation. Journal of Agronomy and Crop Science 193:63-73.

Wang P, Grimm B. 2016. Comparative analysis of light-harvesting antennae and state transition in chlorina and cpSRP mutants. Plant Physiology 172:1519-1531.

Wang Y, Zheng W, Zheng W, Zhu J, Liu Z, Qin J, Li H. 2018. Physiological and transcriptomic analyses of a 
yellow-green mutant with high photosynthetic efficiency in wheat (Triticumaestivum L.). Functional \& Integrative Genomics 18:175-194.

Wang Y, Li Y, Zhang H, Zhai H, Liu Q, He S. 2017. A soluble starch synthase I gene, IbSSI, alters the content, composition, granule size and structure of starch in transgenic sweet potato. Scientific Reports. 7-2315.

Wu ZM, Zhang X, He B, Diao LP, Sheng SL, Wang JL, Guo XP, Su N, Wang LF, Jiang L, Wang CM, Zhai HQ, Wan JM. 2007. A chlorophyll-deficient rice mutant with impaired chlorophyllide esterification in chlorophyll biosynthesis. Plant physiology 145:29-40.

Zhang F, Luo X, Hu B, Wan Y, Xie J. 2013. YGL138(t), encoding a putative signal recognition particle 54 kDa protein, is involved in chloroplast development of rice. Rice (NY) 6:7. https://doi.org/ 10.1186/1939-8433-6-7.

Zhang H, Li J, Yoo JH, Yoo SC, Cho SH, Koh HJ, Seo HS, Paek NC. 2006. Rice Chlorina-1 and Chlorina-9 encode ChlD and ChlI subunits of Mg-chelatase, a key enzyme for chlorophyll synthesis and chloroplast development. Plant Molecular Biology 62:325-337.

Zhang N, Zhang HJ, Zhao B, Sun QQ, Cao YY, Li R, Wu XX, Weeda S, Li L, Ren S, Reiter RJ, Guo YD. 2014. The RNA-seq approach to discriminate gene expression profiles in response to melatonin on cucumber lateral root formation. Journal of Pineal Research 56:39-50.

Zhong X M, Sun SF, Li FH, Wang J, Shi ZS. 2015. Photosynthesis of a yellow-green mutant line in maize. Photosynthetica 53:499-505. 


\section{Figure 1}

Phenotypic characteristics change of yellow-green leaf mutant plants

The letter A indicated two yellow-green leaf mutant plants and a normal green leaf plant at the same age. Maize plants in this image were at the five-leaf stage. The letter B showed the contents of chlorophyll $a$ and chlorophyll $b$ in the normal green leaf inbred line and the yellow-green leaf mutant. The letter $\mathrm{C}$ showed the contents of eight carotenoid compounds including neoxanthin, violaxanthin, capsanthin, zeaxanthin, $\beta$-cryptoxanthin, $a$-carotene, $\beta$ carotene and lutein. Small letters $a$ and $b$ above the columns indicate differences between the yellow-green leaf mutant and the normal green leaf inbred line at $P<0.05$, according to least significant difference (LSD) tests. FW is the abbreviation of the fresh weight. 
A

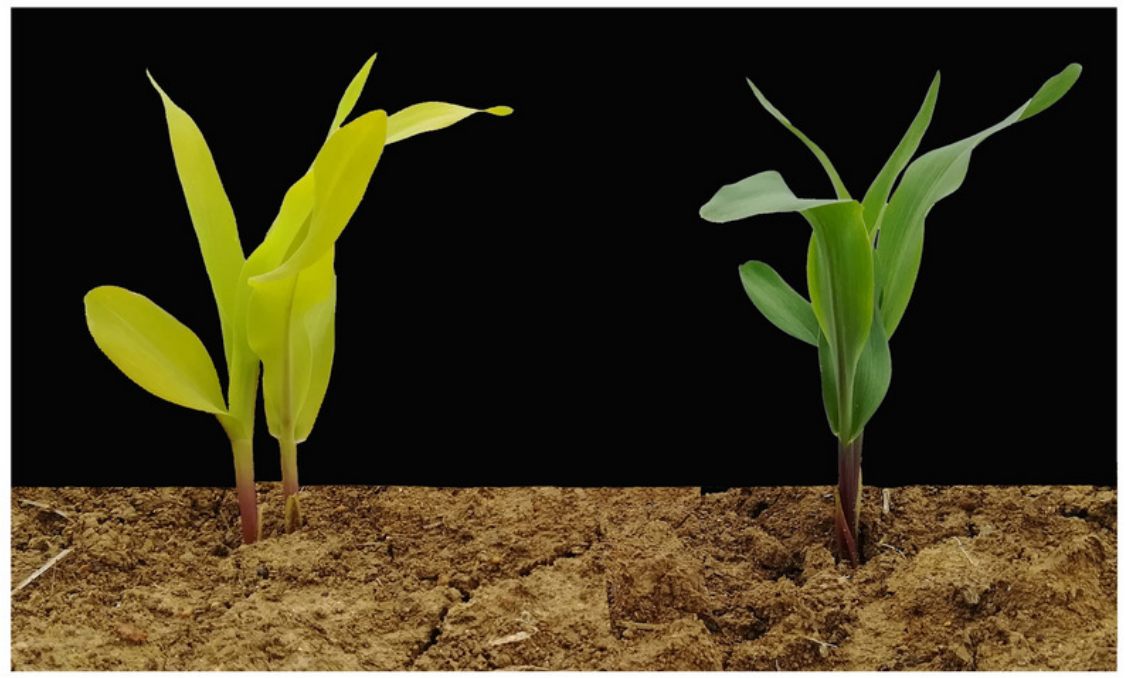

B
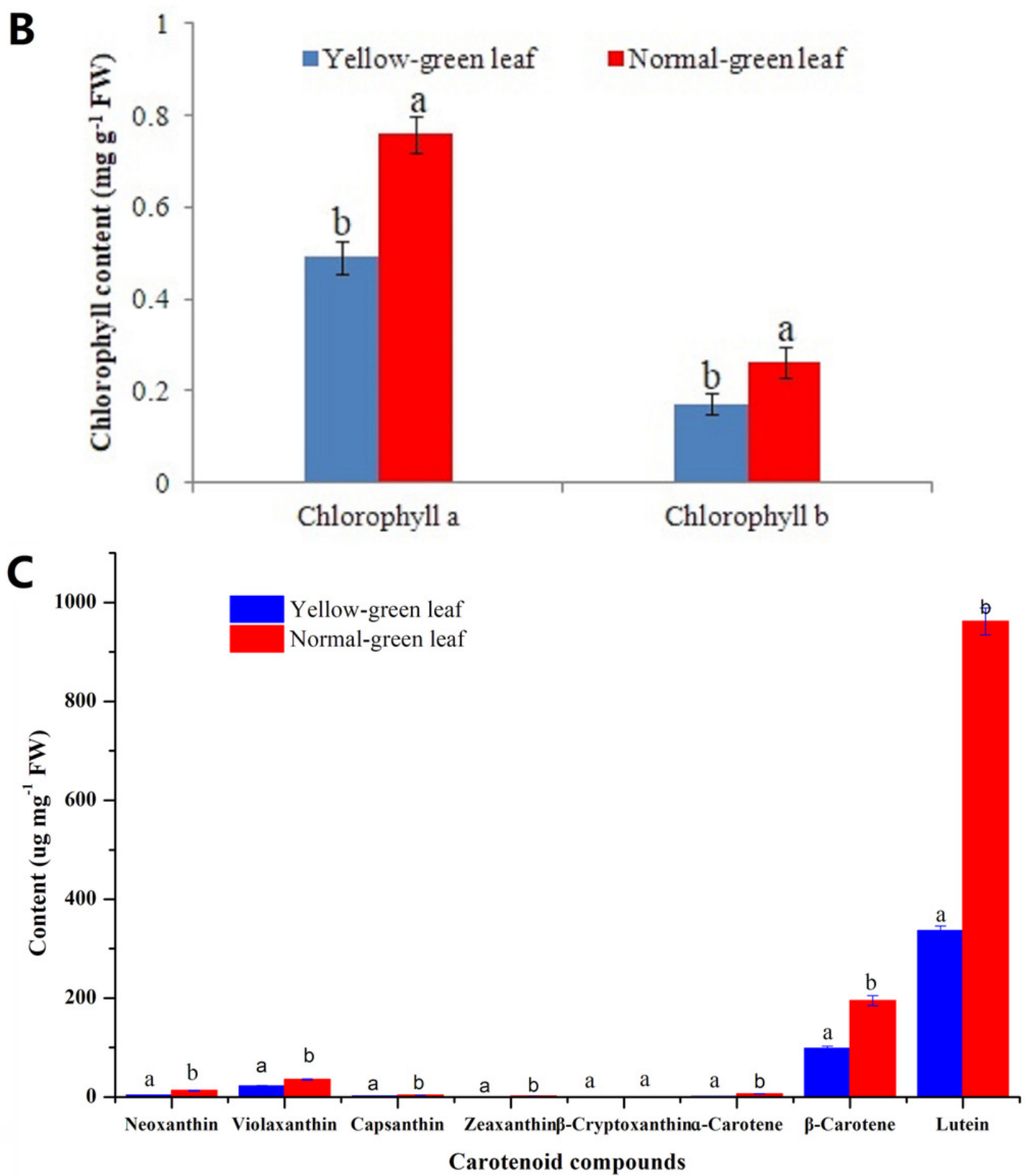


\section{Figure 2}

Chlorophyll fluorescence and photosynthesis parameters in the yellow-green leaf mutant inbred line and the normal green leaf inbred line.

The letter A indicated the original chlorophyll fluorescence parameters.

The letter B showed real-time fluorescence yield $\mathrm{Ft}$ in the normal green leaf inbred line and the yellow-green leaf mutant. The point pointed by the arrows indicated the turned on of the actinic light. The letter $\mathrm{C}$ indicated additional chlorophyll fluorescence parameters of the normal green leaf inbred line and the yellow-green leaf mutant. Small letters $a$ and $b$ indicate differences between the yellow-green leaf mutant and the normal green leaf inbred line at $P<0.05$, according to least significant difference (LSD) tests.

The letter $\mathrm{D}$ showed light response curves of net photosynthesis in the yellow-green leaf mutant and the normal green leaf inbred line. $\mathrm{Pn}$ is the net photosynthesis rate. The light response curves were measured at nine PAR levels $\left(0,50,100,200,400,800,1200,1600\right.$ and $2000 \mu$ molphotons $\left.\cdot \mathrm{m}^{-2} \cdot \mathrm{s}^{-1}\right)$. The dark respiration rate is defined as the $\mathrm{Pn}$ value when the light response curve intersects the $\mathrm{Y}$-axis. The measurement was performed for three times. The error bars represented the standard errors. 

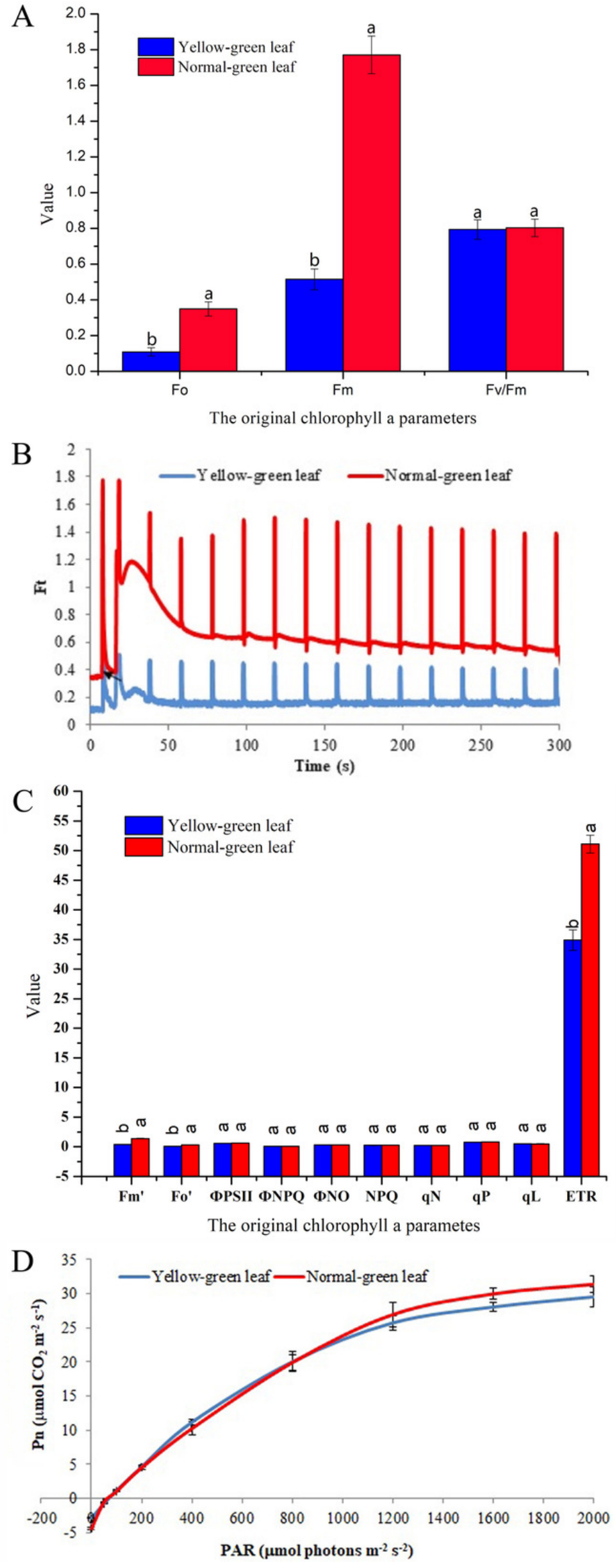


\section{Figure 3}

Differentially expressed genes and its enriched metabolic pathways between the yellowgreen leaf mutant and the normal green leaf inbred line.

The letter A indicated differentially expressed genes between the yellow-green leaf mutant and the normal green leaf inbred line. Differentially expressed genes were selected by $q$ value $<0.005 \& \mid \log 2$ (fold change) $\mid>1$. The $X$ axis indicates gene expression changes in different samples, and the $Y$ axis indicates the significant degree of gene expression changes. Scattered points represent each gene, the red dots represent differentially upregulated genes, the green dots represent differentially down-regulated genes, and the blue dots represent no significant difference gene. YGL, yellow-green leaf mutant; NGL, normal green leaf inbred line; $-\log 10$ (padj), the corrected $p$-value $($ padj $<0.05)$. The letter B showed the pie chart of enriched metabolic pathways of genes differentially expressed in the yellowgreen leaf mutant and the normal green leaf inbred line. The pie chart was generated by submission of the differentially expressed genes to the online Mercator sequence annotation tool (http://www.plabipd.de/portal/mercator-sequence-annotation). 
A

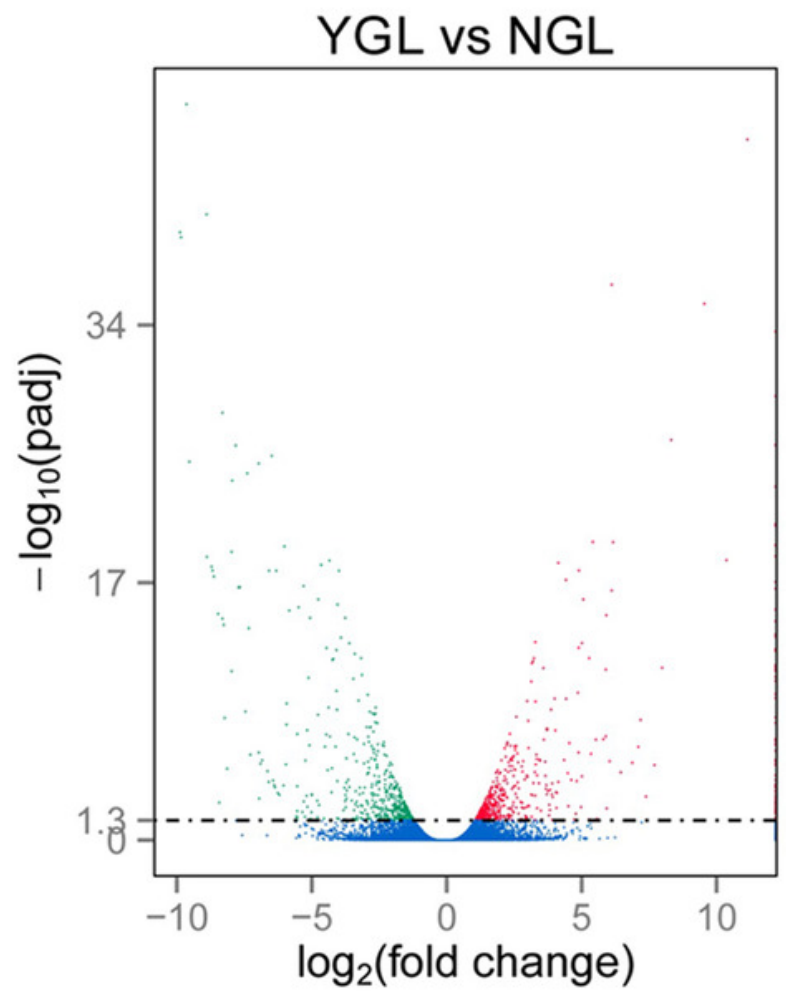

Differential Expressed Genes ( 1122 )

up regulated: 536

down regulated: 586

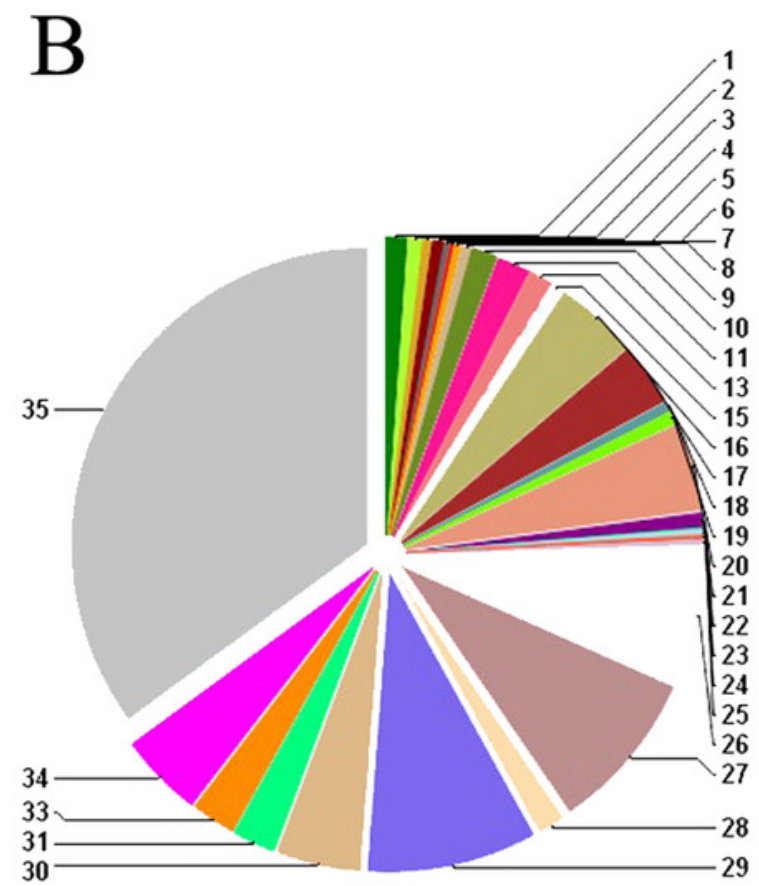

\begin{tabular}{|c|c|c|c|c|c|}
\hline \multirow{2}{*}{$\begin{array}{l}\text { Pathway } \\
\text { 1-PS }\end{array}$} & \multicolumn{2}{|c|}{ mber Percentage } & Pathway & \multicolumn{2}{|c|}{ Gene number Percentage } \\
\hline & 14 & $1.11 \%$ & $\begin{array}{l}\text { 2- major } \mathrm{CHO} \\
\text { metabolism }\end{array}$ & 9 & $0.79 \%$ \\
\hline $\begin{array}{l}\text { minor } \text { CHO } \\
\text { metabolism }\end{array}$ & 4 & $0.40 \%$ & 4. glycolysis & 8 & $0.64 \%$ \\
\hline - 5- fermentation & 4 & $0.32 \%$ & $\begin{array}{l}\text { 6- gluconeogenesis / } \\
\text { g/yoxy/ate cycle }\end{array}$ & 1 & $0.08 \%$ \\
\hline $7 \cdot O P P$ & 2 & $0.16 \%$ & $\begin{array}{l}\text { 8CA / org } \\
\text { transformation }\end{array}$ & 4 & $0.32 \%$ \\
\hline $\begin{array}{l}\text { 9. mitochondrial } \\
\text { electron transport / } \\
\text { ATP synthesis }\end{array}$ & 7 & $0.56 \%$ & ㅁ.ce/l wall & 17 & $1.35 \%$ \\
\hline 11 - lipid metabolism & 19 & $1.75 \%$ & $\begin{array}{r}13 \text { - amino acid } \\
\text { metabolism }\end{array}$ & 16 & $1.35 \%$ \\
\hline 15 - metal handling & 6 & $0.48 \%$ & $\begin{array}{l}16 \cdot \text { secondary } \\
\text { metabolism }\end{array}$ & 52 & $4.45 \%$ \\
\hline 17 - hormone metabolism & 40 & $3.34 \%$ & $\begin{array}{l}18 \cdot \text { Co-factor and } \\
\text { vitamine metabolisn }\end{array}$ & $n^{6}$ & $0.48 \%$ \\
\hline 19 -tetrapyrrole synthesis & is 7 & $0.64 \%$ & $20 \cdot-s t r e s s$ & 55 & $4.69 \%$ \\
\hline $21-$ redox & 10 & $0.79 \%$ & $\begin{array}{c}22 \text {-polyamine } \\
\text { metabolism }\end{array}$ & 1 & $0.08 \%$ \\
\hline $\begin{array}{c}23-\text { nucleotide } \\
\text { metabolism }\end{array}$ & 4 & $0.32 \%$ & $\begin{array}{c}24 \text { - Biodegradation of } \\
\text { Xenobiotics }\end{array}$ & 2 & $0.16 \%$ \\
\hline $25 \cdot$ - C1-metabolism & 3 & $0.24 \%$ & 26 - misc & 87 & $7.15 \%$ \\
\hline $27 \cdot R N A$ & 106 & $8.82 \%$ & $28-D N A$ & 13 & $1.51 \%$ \\
\hline $29-$ protein & 106 & $9.22 \%$ & 30 -signalling & 54 & $4.53 \%$ \\
\hline $31 \cdot c e / l$ & 26 & $231 \%$ & = 33 -development & 27 & $238 \%$ \\
\hline - 34 -transport & 53 & $4.53 \%$ & $35 \cdot$ not assigned & 328 & $35.06 \%$ \\
\hline
\end{tabular}




\section{Figure 4}

Comparative expression analysis of differentially expressed genes involved in chlorophyll metabolism between the yellow-green leaf mutant and the normal green leaf inbred line.

\section{Chlorophyll $b$}

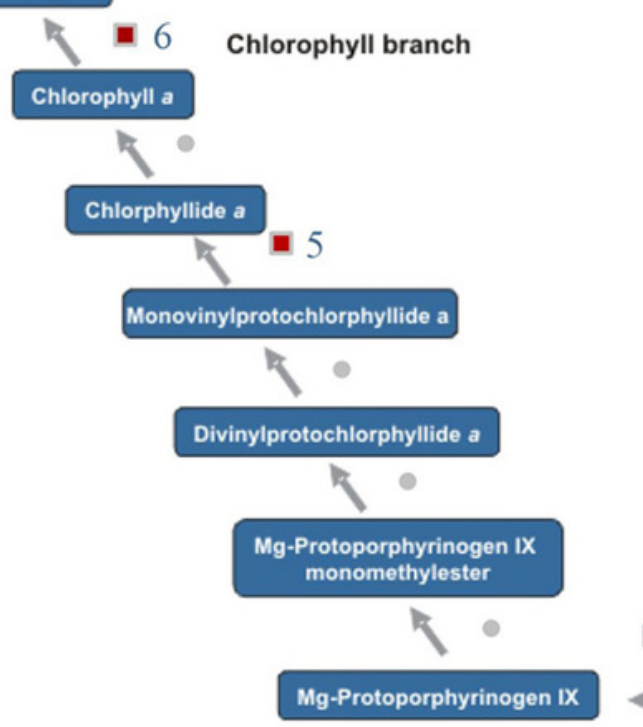

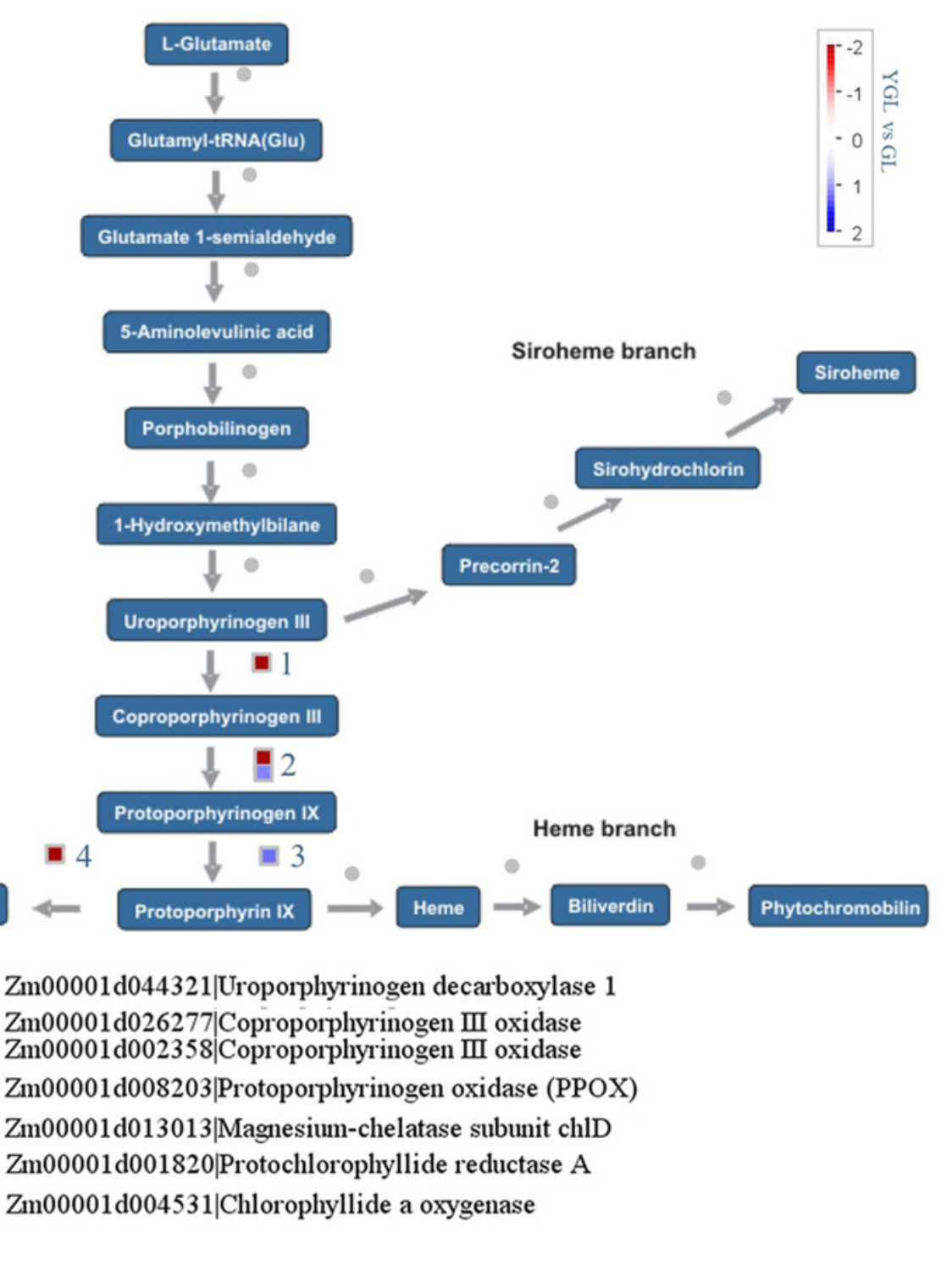


Figure 5

Comparative expression analysis of differentially expressed genes involved in photosynthesis light reactions and carbon reactions between the yellow-green leaf mutant and the normal green leaf inbred line. 

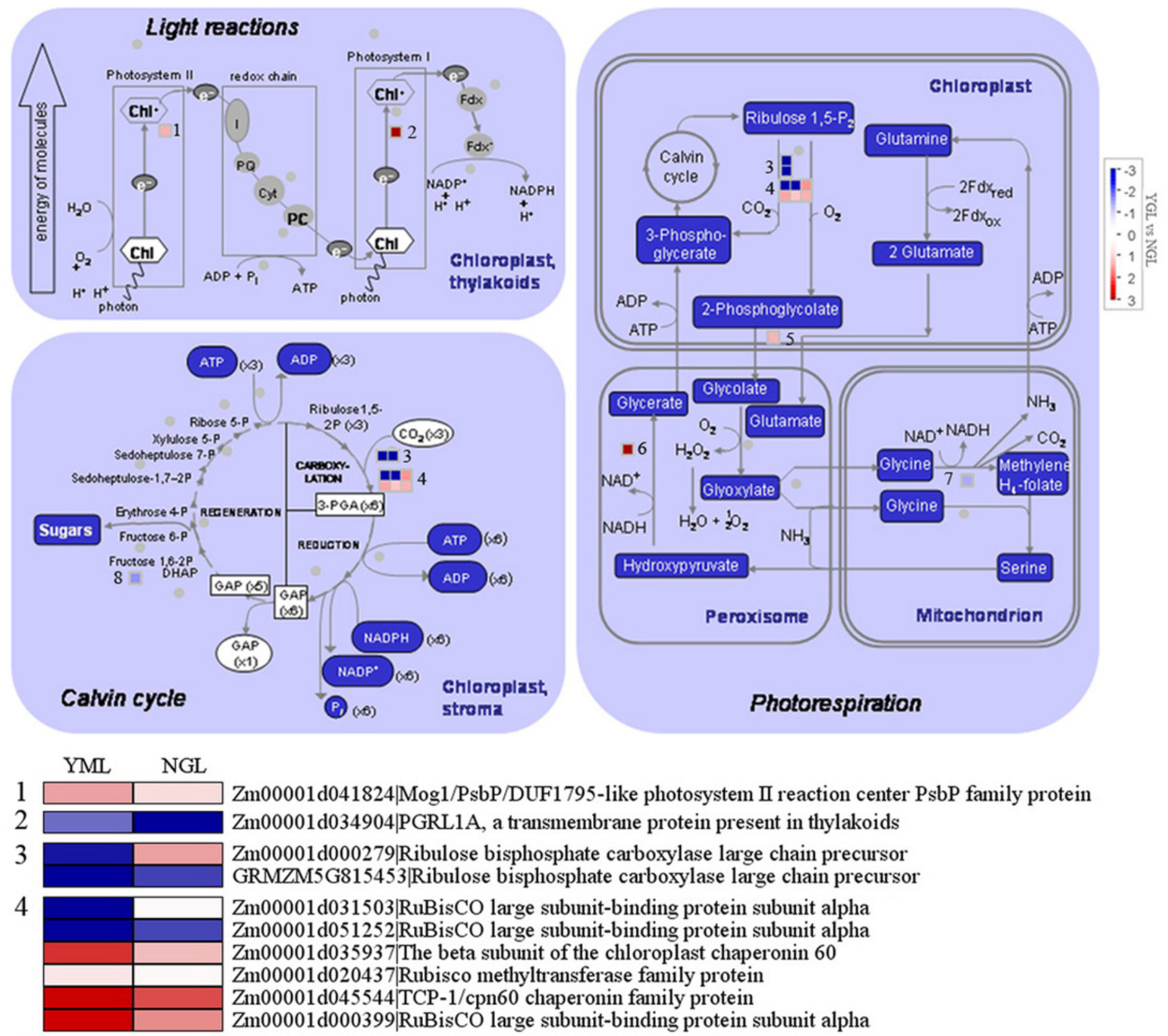

Zm00001d041824|Mog1/PsbP/DUF1795-like photosystem II reaction center PsbP family protein Zm00001d034904|PGRL1A, a transmembrane protein present in thylakoids

ร

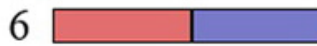

Zm00001d000279|Ribulose bisphosphate carboxylase large chain precursor GRMZM5G815453|Ribulose bisphosphate carboxylase large chain precursor

$\mathrm{Zm} 00001 \mathrm{~d} 031503 \mid \mathrm{RuBisCO}$ large subunit-binding protein subunit alpha $\mathrm{Zm} 00001 \mathrm{~d} 051252 \mid \mathrm{RuBisCO}$ large subunit-binding protein subunit alpha $\mathrm{Zm} 00001 \mathrm{~d} 035937$ The beta subunit of the chloroplast chaperonin 60 Zm00001d020437|Rubisco methyltransferase family protein $\mathrm{Zm} 00001 \mathrm{~d} 045544 \mid \mathrm{TCP}-1 / \mathrm{cpn} 60$ chaperonin family protein $\mathrm{Zm} 00001 \mathrm{~d} 000399 \mid \mathrm{RuBisCO}$ large subunit-binding protein subunit alpha

\section{7}

8 Zm00001d034887|2-Phosphoglycolate phosphatase 2 (PGLP2)

Zm00001d014919|Glycerate dehydrogenase

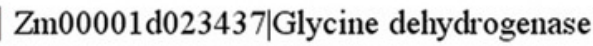
Zm00001d040084|Aldolase superfamily protein 


\section{Figure 6}

\section{Comparative expression analysis of differentially expressed genes in the tricarboxylic acid cycle between the yellow-green leaf mutant and the normal green leaf inbred line.}

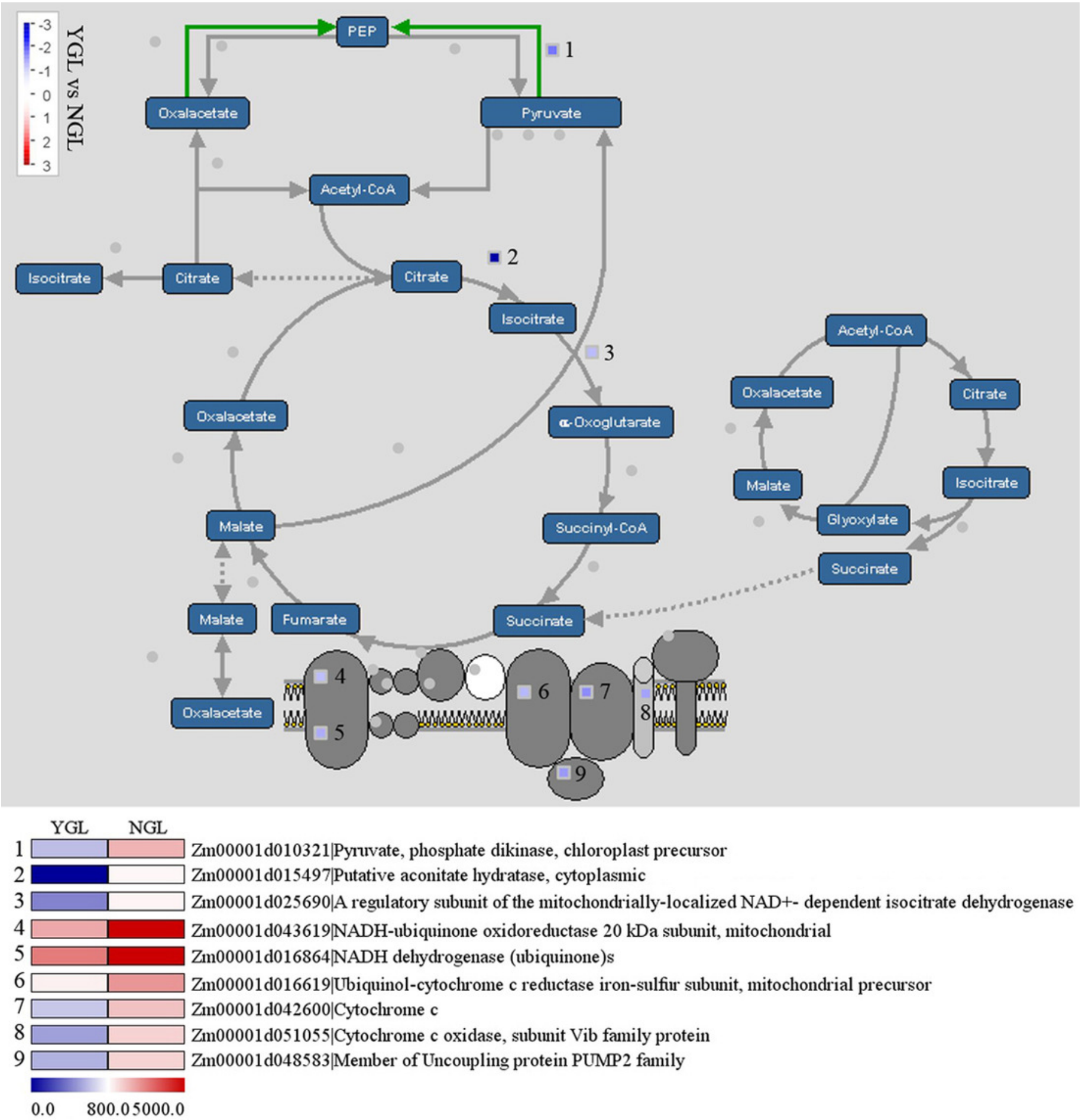

PeerJ reviewing PDF | (2020:06:49778:3:0:NEW 6 Nov 2020) 


\section{Figure 7}

Comparative expression analysis of differentially expressed genes involved in sucrose to starch conversion between the yellow-green leaf mutant and the normal green leaf inbred line.
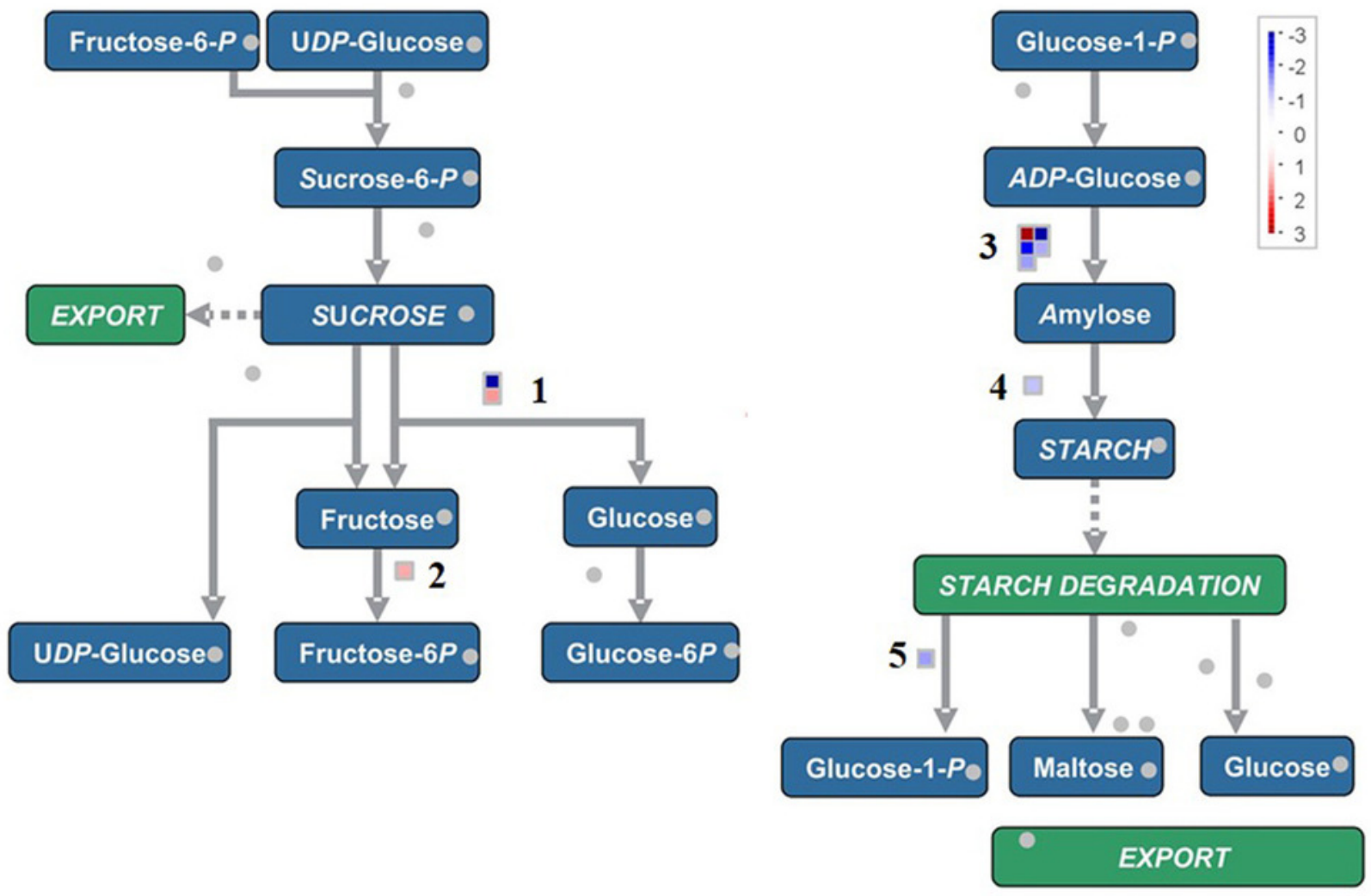

1

2

3

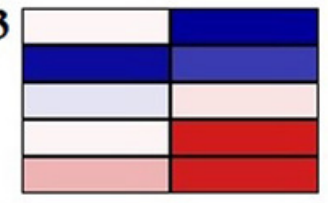

4

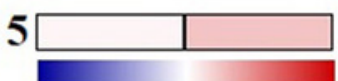

0.0

400.06000 .0

$\mathrm{Zm} 00001 \mathrm{~d} 025354 \mid$ Beta-fructofuranosidase, insoluble isoenzyme 2 precursor $\mathrm{Zm} 00001 \mathrm{~d} 025943$ Beta-fiuctofuranosidase 1 precursor,glycosyl hydrolases family 32 protein $\mathrm{Zm00001d033181|Fructokinase-like} \mathrm{protein,} \mathrm{a} \mathrm{member} \mathrm{of} \mathrm{the} \mathrm{pfkB-carbohydrate} \mathrm{kinase} \mathrm{family}$

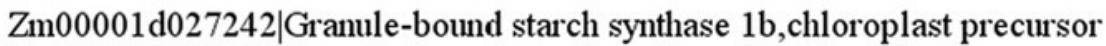

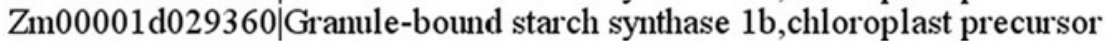
Zm00001d002256|Soluble starch synthase 3, chloroplast precursor $\mathrm{Zm} 00001 \mathrm{~d} 019479 \mid$ Granule-bound starch synthase 1b,chloroplast precursor Zm00001d045261|Soluble starch synthase 1, chloroplast precursor Zm00001d003817|1,4-alpha-glucan branching enzyme IB, chloroplast precursor Zm00001d034074|Alpha-1,4 glucan phosphorylase L isozyme, chloroplast precursor 